\title{
Physical and Behavioral Markers Help Identify Written Language Disability (WLD) Related to Attention Deficit Hyperactivity Disorder (ADHD)*
}

\author{
Rowe A. Young\#, Benson E. Ginsburg, Dawn Bradway \\ Department of Psychology Program/Behavioral Neuroscience, University of Connecticut Storrs, \\ Connecticut, USA \\ Email: "Roweyk@gmail.com
}

Received October $13^{\text {th }}, 2011$; revised November $12^{\text {th }}, 2011$; accepted December $13^{\text {th }}, 2011$

\begin{abstract}
Rotation, its physiological processing, as well as how this lateralized direction of movement is sensed, is regularly taken for granted and little appreciated when studying how learning develops. Preference for direction of rotational movement and how one processes this information has been found to differ greatly from one individual to another. This report discusses how bimanual rotational hand use preference and reversed direction of body part and movement sensation detection may be utilized to help identify certain currently unrecognized classroom learning difficulties. What we label Reversed Positioning Sensation (RPS) is evidenced in our research and is found in many children and adults presently identified as "learning disabled" and/or "hyperactive" or just those considered "bright underachievers". Easily administered tests to help identify individuals who may be at risk are discussed. When RPS occurs without the necessary sensory-motor accommodation or compensation, we theorize that written language disability (WLD), as well as compromised organizational skills often identified as ADHD, result. In addition, this paper discusses a summary of our findings examining families with biologically related family members reporting primary difficulties related to organization and written language skills. Case studies from four of the many families we have studied demonstrate how to determine if an individual is able to sense his/her own body extremities in correctly functioning or maladaptive directional orientation. In some cases, just changing a writing hand position has been shown to help overcome many of the problems of RPS. This change is meant is to redirect the writing hand movement to sense a top/down orientation.
\end{abstract}

Keywords: Rotation; WLD; Written Language Disability; Motor Learning; Proprioception; Kinesthesia; Reversal; Directional Movement Sense; Learning Disability; Attention Deficit Hyperactivity Disorder; ADHD

\section{Background}

Members of a former research team from the University of Connecticut Biobehavioral Research Laboratory worked for many years to gain more understanding of academic underachievement involving motoric behavior from a bio-behavioral perspective. Past results have shown comparative motor patterns relate to academic underachievement (Bradway, 1999, 2003; Carter, 1977, 1982; Ginsburg, 1980; Sadick, 1978; Young, 1987, 1989, 1993).

From accumulated evidence in various fields, it is commonly accepted that academic achievement is dependent upon the proper neurological integration of visual, motor and aural capabilities (Wolf, 1996). However, as Lyon, (1996) noted, "Currently, there are no universally accepted tests, assessment batteries nor standards for identifying children with WLD (written language disability)" (Lyon, 1996). More recently, Katusic and colleagues stated that, "Since children with WLD are clearly a heterogeneous group, additional research is needed to identify specific interventions that target the specifics of writing problems" (Katusic, 2009)

In aiding this identification, it is suggested that a complex

"Conflicts of Interest: The authors have no conflicts to report.

${ }^{\#}$ Corresponding author. (yet uniquely individual) kinesthetic movement integration process is directly involved. This integration of the sense of movement in symbolic comprehension, we hypothesize, is necessarily aided by the feeling for proper directional orientation in fine motor as well as gross movement behavior. This might relate to the same spatial mapping in the hands as proposed by LeDoux and colleagues, who stated that the "manipulo-spatial function is neither motor nor perceptual, per se, but rather is more appropriately viewed as the mechanism by which a spatial context is mapped into the perceptual and motor activities of the hands" (LeDoux, 1977).

As a confounder to this mechanism, the authors propose motoric directional inverted sensation, which we label reversed positioning sensation (RPS). The authors further suggest that these patterns have their origins in the interconnected hemispheric proprioception emanating from the parietal lobes which sense where one's body parts are spatially positioned (Adams, 1997; Cook, 2006).

One early participating female adult subject became aware of specific unusual lateral body movement sensations as a result of the project. This information was described to us. The description of the reversal of specific movement sensations was later found to match an unusual arm and hand position. We then were able to identify similar physical characteristics in many of 
the other individuals with written language difficulties (Young \& Ginsburg, 1993).

From our findings, RPS is further defined as the inverted reversal of the directional sense of feeling for movement occurring in both the fine and the large motor control of the body. This condition may occur laterally and differently between an arm, hand and/or fingers and the leg, foot and/or toes. This may or may not occur in the same way in one or all of the limbs of the body, with or without the presence of movement. It may also include a physical appearance as well as the sensory-motor manifestation. To better understand the effects of RPS, it might, e.g., be compared with looking through a transparent piece of writing material from the backside and trying to read what has just been written from the opposite side. This would be representative of a similar comprehensive effect, only visually.

The purpose of this research has been to both explore the development of methods both to help identify and to remediate the potential maladaptive affects of RPS. We theorize that this invisible reversal of movement sensory difficulty confounds basic learning and overall organization resulting in ADHD. Early academic skills are theorized to be the most impacted. We suspect those affected eventually learn to cope by using other learning capabilities. This is not without the trauma of the misunderstanding of why the learning of basic skills is so difficult.

Through observation of many subjects, our research has identified that in a significant portion of a population, rotational movement patterns differ from commonly expected behavioral patterns. We've explored this finding by testing over one thousand subjects coming from several (elementary through college) school populations, as well as an opportunistic sample of volunteers and several multi generational families (Young, 2006, 2008).

The test we developed for this research is called the YoungGinsburg Lateral Direction Assessment and Spatial Test (YGLD). After having seen these patterns commonly present in family members with a diagnosed learning disability (LD) most often found to be WLD because of the motoric aspects of the identification. We now theorize that RPS occurs for genetic reasons. In order to measure these observations in a controlled behavioral manner, we set up experiments, to see if we could behaviorally identify WLD family members compared to non-WLD members based upon these criteria.

\section{Methods}

\section{Participants}

The original opportunistic pool of several family groups were selected after we were made aware that there were one or more members in a family who had been diagnosed with a learning disability. Upon reviewing both school records and self reports, problems with first learning to read, spelling, and penmanship appear to be the most common factors of academic difficulties reported. The label WLD, and what it implies today, might be more appropriate.

We administered our test battery to all of the family members who were available and agreed to participate in our study. Participants from these families were tested in different parts of the United States. These family members were generally from well educated backgrounds.

This present report has been formed around four selected members from four family groups taken out of the many fami- lies we have tested. The four case studies being focused on were selected as a result of experimental remediation to try and reverse the directional confusion identified. Only one of these four families had enough participants $(n=64)$ to perform a statistical analysis.

\section{Study Design}

The subtests administered included: direction preference for different types of rotation of both fine and large motor movements, visual and manual interactive hand use and asymmetrical arm and movement identification, using a combination of paper and pencil testing, interview and observation techniques. The family members used in this report were also all tested for reversed body sensations in their arms and hands.

Only the YGLD tests relating to this present report will be described herein. Participants also filled out a questionnaire regarding their academic achievement where no school records were available. While much information was self-report, school records and testing were provided wherever possible.

\section{Test 1. Fine Motor Bimanual Rotation Test}

A small bottle with a one inch in diameter cap is used in this test. The participant is first asked to unscrew the lid from the bottle. The examiner then marks what method that was used. Next, the subject is asked to watch as each of seven methods to unscrew the lid of the bottle is demonstrated.

Following the demonstration, the subject tries each of the methods. Only the technique(s) that appear natural and comfortable to the subject and that the subject says they would use are marked. Three possible designations are based upon the answers that are given for the test items having to do with fine motor bimanual rotation:

1) Exclusive Left Hand Top: counterclockwise movement with no bottom right hand movement or right hand movement in tandem with the top left hand.

2) Bottom Turn Hand: hold the bottle in a stationary manner with the top hand and turn with the bottom hand. This can be by using either hand and in conjunction with any other turning methods that are found comfortable.

3) Exclusive Right turning hand on the top with a stationary left or tandem left hand on the bottom, or Right and Left hand on Top: with a stationary hold or tandem movement on the bottom.

These three groups are used to form the analysis of our primary data of Family D. Exclusive Right and Either top (both exclusive right and both right and left top) have been combined, as the results in our data produce the same effect between the division of WLD and non-WLD.

\section{Test 2. Arm and Hand Positioning Test (For Asymmetry and/or an Arm Shifting Position)}

Questions to determine how the arms compare to one another were used to determine if the two arms are alike or different. The examiner first tells the participant: "Stand up straight, let your arms and hands hang loosely by your sides. Let's look at both of your arms in a relaxed position". The first part of the identification is looking at the position of the relaxed arms. The side of the arm (below the elbow) that matches the back of the hand is observed. The backs of some arms and hands are facing 
forward, while some are facing sideways. In some cases, one arm faces in one direction and the other arm in another direction. Other cases are observed to be in non-alignment, i.e., an outward facing back of the arm with a forward facing hand. Occasionally an arm and/or hand will naturally position itself in a backward facing position.

Next, the participant is asked, "Stretch your arms down at your sides as stiffly, as you can". Some subjects are found to have a different positioning of the arms in a stiffened position. We were also looking for a shift of arm position that does not affect the position of the hand. The participant's so defined different arm position characteristics when compared to the other arm are classified, as being asymmetrical.

The four case studies discussed were also asked for perception of arm, hand and finger positions. This was done by having the subject stretch out their arms from the elbows to the hands, with palms rested palms on a table. Subjects were asked to concentrate on the feel of both arms and hands with their eyes closed. They then were asked to compare if their hands felt as if they are both facing downward or upward. The subjects were then asked if one hand felt like it was facing downward while the other felt like it was facing upward. Lastly, subjects were asked to compare their arms to one another. Could one lower arm feel as if it was facing downward and the other upward? If differences were found, they were then asked to explain the difference.

As some of the family study D members were not tested for perception of body part position characteristics; we were not able to compare this variable in this family statistically.

Using the following test sequence, the arm hand/eye dominance test identifies which eye is dominant when sighting a target using the arm hand/eye combinations described:

\section{Test 3. Arm Hand/Eye Dominance Test}

Begin each test with arm being used out stretched

Circle - Find the eye that stays aligned to the (ten feet away) target and in the circle that the subject has made with their thumb and index finger.

(Start with both eyes open, find the target, set position, and then close one at a time.)

1) Using the right arm; the eye on the target is (mark one):

a) right __ b) left___ c) neither

2) Using the left arm; the eye on the target is (mark one)
a) right b) left c) neither

Point-Repeat the procedure above in but now, point to the target

Now use the index finger and line it up to the target

1) Using the right arm; the eye on the target is (mark one):

a) right b) left__ c) neither

2) Using the left arm; the eye on the target is (mark one)

a) right b) left c) neither

\section{Results}

\section{Case Studies}

Long term results of this 25-year project, are the results of remaining involved with some of the families with identified LD members, as well as with other family members demonstrating the characteristics of RPS.

Following are excerpts from the information collected from these four cases studies from the four different participating families which are discussed in the present report.

\section{Family A (n = 7), Case Study 1}

An adult male was referred to us by his former high school teacher. She could not understand how such an "intelligent" student could not learn to read and write at grade level. This subject also brought us his three oldest children for participation in the study. These four family members, all with reading and writing problems, also displayed inconsistency of preferred use of the bottom hand turning in the lid unscrewing activity designed for our experiment. The father both used and inverted his left hand when he wrote. The father and his sons all displayed unusual asymmetrical rotated arm positions, evidenced when the arms are stretched out, as illustrated in Figures $\mathbf{1}$ and 2. The four family members all displayed as well as reported inverted directional bi-manual movement preference.

The seven-year-old son wrote with his right arm-inverted hand position, in a claw like grasp. He expressed feelings of reversal of direction sensation in his right arm and hand.

The six year old son also had feelings of the right arm and hand feeling upward when compared to the left arm and hand when both were in a down-ward position.

The 8-year-old expressed feelings of inverted feelings in different areas of his extremities.

At the time we first saw this family, the youngest two children were not yet identified as learning disability LD; however, they were all identified LD by their school the following year. There were also two daughters in this family who reported inverted sensations in their arms and displayed the inverted bimanual rotation characteristic. There was no asymmetry seen in their arms.

All five children were found to be of normal intelligence and diagnosed by their school psychologist as having LD. The more standard experimental remediation we attempted with these family members did not appear to have any lasting effect.

The only other member tested of this family was the mother and found to have none of the RPS symptoms. She also was found to have no history of any learning difficulties.

\section{Family B (n = 7), Case Study 2}

Another target subject from a family we were opportunistically invited to test. This was an eight year old gifted female with written language problems. Her arms displayed the asymmetrical arm positions we have described (Figure 3).

Case Study 2, as well as her mother, father, and brother, all demonstrated and reported the inverted preference for the bimanual rotation activity. There however were also no asymmetries observed. They all however, reported some written language difficulties. Three other members of this family were tested and did not have any of the characteristics of asymmetry or bi-manual inversion preference.

Of her own choice, Case Study 2 reportedly changed from writing with her left hand to using her right hand in kindergarten. She was tested to have a full scale IQ of 139 on a revised edition of the Wechsler Intelligence Scale for Children (WISC-R). The Stanford Diagnostic reading test revealed her total reading capabilities at less than a 50th percentile rank. On the Test of Written Language (TOWL) she scored at the 50th percentile in spelling and at the ninth percentile in handwriting. She had a total written language score of 110 . She was placed 


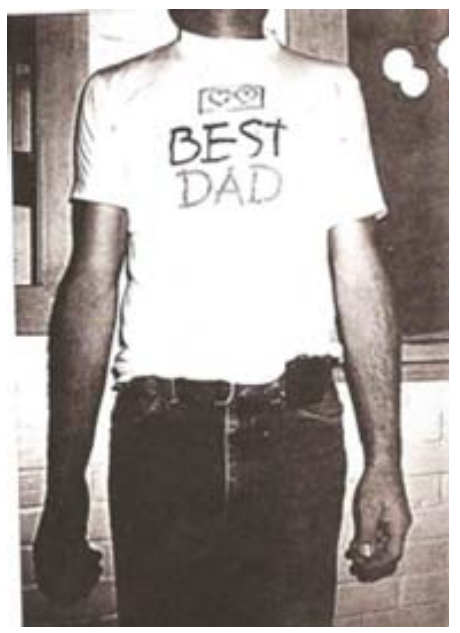

(a)

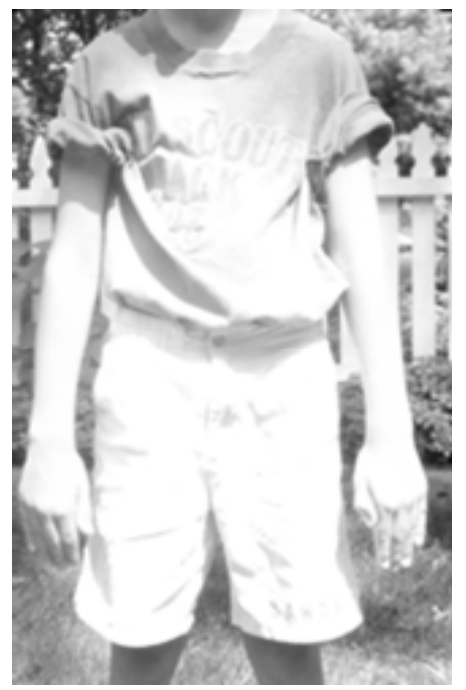

(b)

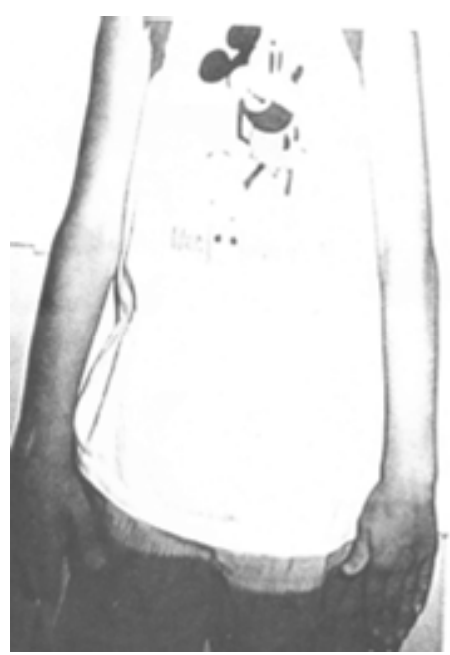

(c)

Figure 1.

Family A, Case Study 1: asymmetrical stretched arm positions in a father (a) and his sons aged 7 years (b) and 6 years (c).

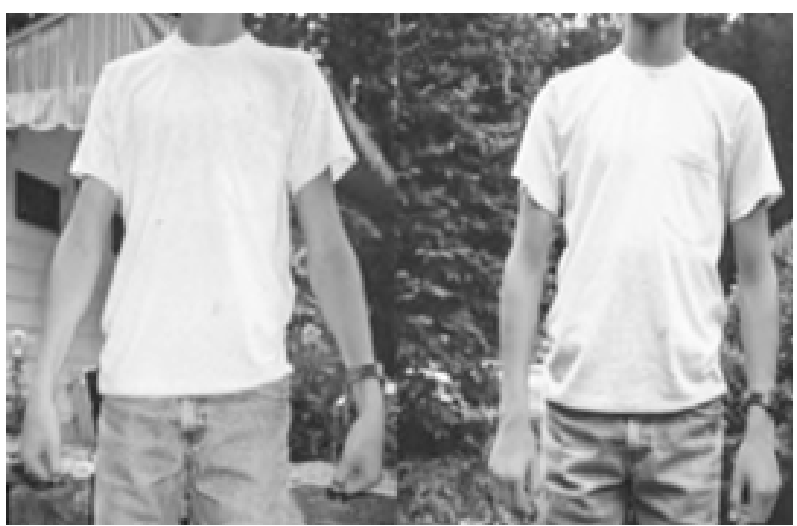

(a)

(b)

Figure 2.

Family A, 8-year-old male in stretched (a) and then relaxed (b) arm position.

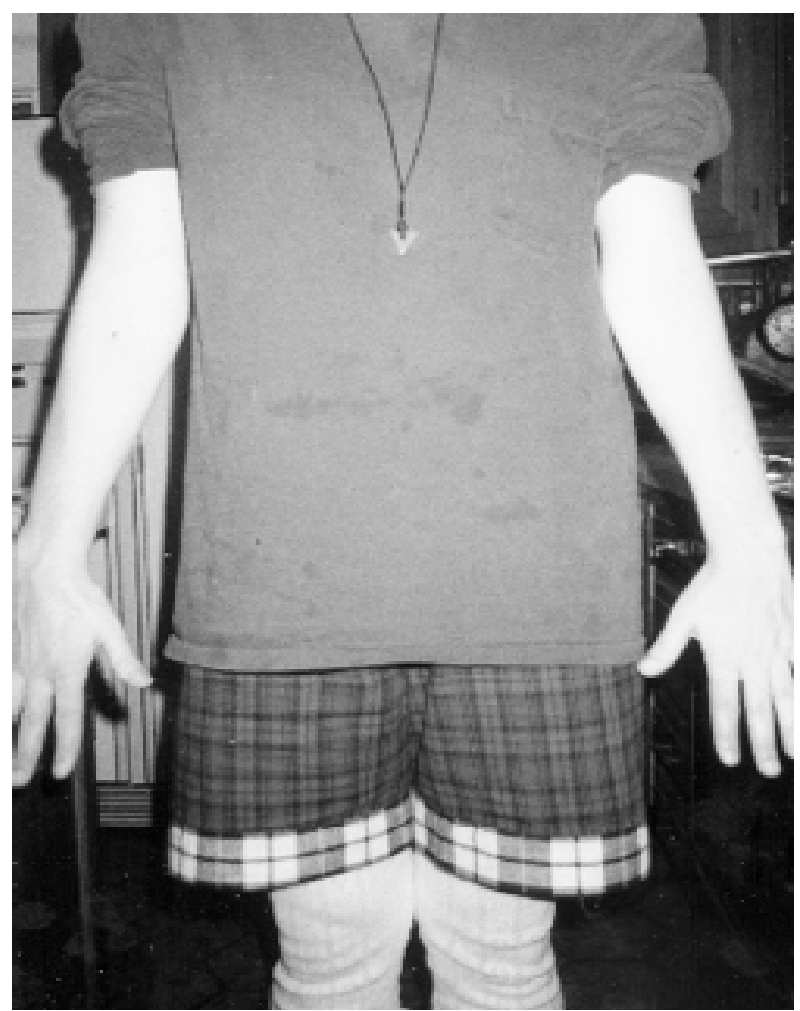

Figure 3.

Family B, Case Study 2: female in a stretched arm position displaying an asymmetrical position in both arms.

in the high performance groups in school, where her grades were barely average. The head of the program considered dropping her from the program when we started seeing her, even though she worked very hard at her studies.

As a remedial experiment she was encouraged to place her pencil between her second and third fingers to change the position of her hand when writing. After having used the position for four years she reported to us that she was aware of the reverse feeling in her hand and arm when she used her old hand writing position. She had now risen to the top 20th percentile of her class. This student was now achieving all A's and B's in- 
stead of predominantly C's.

Today, many years later, she is a school administrator. In a recent correspondence, she told us "It seems, upon reflection, that by using the modified grip for my pen/pencil, my thoughts became more organized and clear and I had to write down the plan for any essay I was creating so as to not lose them. This became my standard practice in college as I had many essay exams/papers to write, [double major in English and psychology]. Overall, the modified hand grip had mechanical and cognitive benefits that I can clearly remember even today, almost 25 years later".

\section{Family C (n = 8), Case Study 3}

A teacher who was aware of our project referred to us a ten-year-old boy. He was identified by his school as being both LD and having ADHD. He was tested to have a full scale IQ of 130/136 on WISC-R. On the Wide Range Achievement Test (WRAT-R), his word recognition was at the 4th percentile and spelling was at the 19th percentile. On the Woodcock Johnson Reading Cluster his total reading score was at the $\left(17^{\text {th }}\right.$ percenttile) 2.0 grade level. We tested him and found him to have both the asymmetrical arm and reversed bimanual opening characteristics. Figure 4 illustrates Case Study 3 with his right arm stretched in a hyper-extended position and Table 1 details his physical and sensory markers that appear to be characteristic of RPS. [A maternal grandmother was the only member of the seven members of his family we tested who demonstrated the inverted bimanual rotation. However, no educational report was available.]

As a remedial experiment, we changed the position of his hand by putting the pencil between his second and middle finger so as to move clockwise and feel a top down sensation.

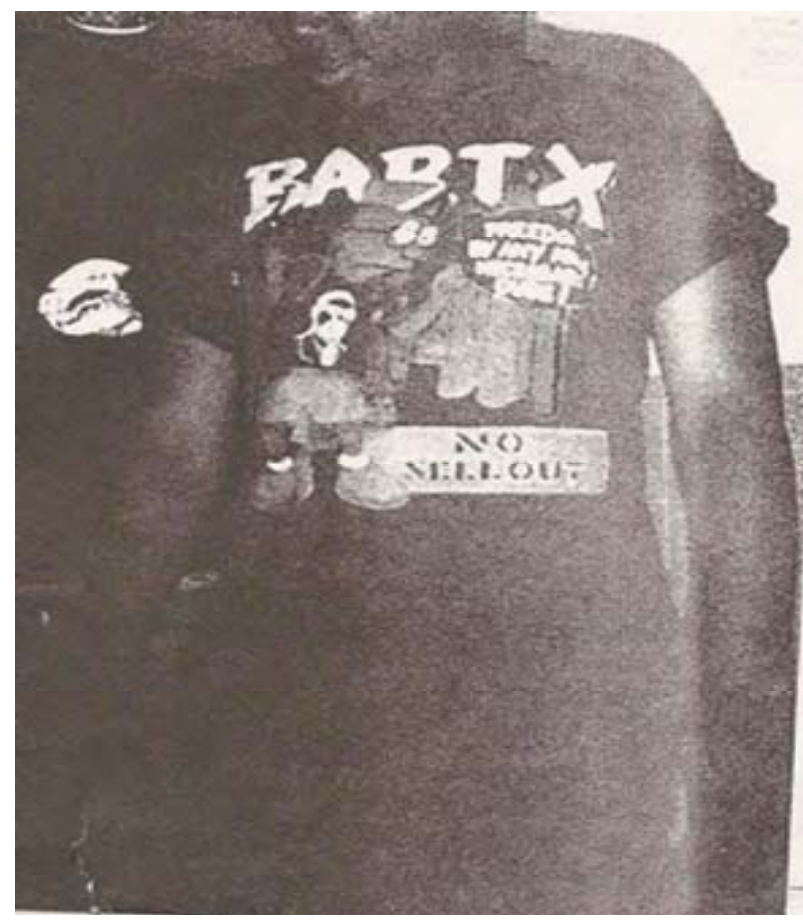

Figure 4.

Family C, Case Study 3: Male with arms in the stretched position displaying right arm asymmetry.
Table 1.

Family C, Case Study 3: Physical and sensory markers characteristic of RPS observed in male with arms in the stretched position displaying right asymmetrical hyper-extended arm.

- Feeling shorter limbs on the right side

- Right hand writing position that follows the hyper-extended turn of the right arm

- Direction confusion with just the right hand

- Using index finger on the left hand to point out direction

- Avoids using the index finger on the right hand

- Names and points to an eye as the one being used when it is not being used

After coming for experimental remediation once a week for a year he was retested on the Woodcock-Johnson Reading Cluster Test. His total reading score was found to be at the $\left(38^{\text {th }}\right.$ percentile) 4.0 grade level. Upon following up his case two years later, we found no change from the last time that we saw him; his reading ability was still at 4.0 grade level and he continued to have trouble with written assignments. We also found that he had not stayed with the changed handwriting position. He again had the direction of a rotation confused. As an example, he identified a clockwise rotation from the bottom of the rotation as going left.

\section{Family D (n = 64), Case Study 4}

There were fifteen cases of learning difficulties related to written language reported to us from this opportunistically found family consisting of four generations. The female in generation one of Family D was tested and was found to not to have the symptoms of RPS. Members from her parent's family $(\mathrm{n}=10)$, who were available also tested, did not have these symptoms. The first generation male member, her husband, and his parent's family were not available for testing.

A generation 2 family member was a gifted pianist and composer, but could not read or write music and had other written language disability symptoms. She was tested and found to be a bimanual inverted rotator with a shifting in the right arm, as illustrated in Figure 5.

As a reportedly presumed result of her own written language struggles, she went on to develop an early on Pre First program for bright underachievers in the school district in which she taught.

A third generation member of this family group (Case 4; one of our first remediation experiments), was a 48-year-old woman with a LD history we call "A.L". Case 4 was A.L. displayed many of the symptoms itemized on the Wender Utah Criteria for Adult ADD (Wender, 1990), i.e., persistent hyperactivity shown by restlessness; the inability to relax, being "always on the go," lack of organization, impulsively, and chronic stress. Her major academic problems were described as the result of reading fatigue, i.e., she bit her nails when reading and had handwriting difficulties, made spelling errors. In spite of these problems, she managed to graduate from college.

We assessed A.L. to be left-handed and left footed and mixed eye dominant (using a left, right, left, left pattern). In spite of her direction confusion difficulties, we found this subject to be perceptually within normal limits. We became more interested in her fine motor laterality difference when she related these experiences. Unknowingly, A.L. taught herself to use a com- 


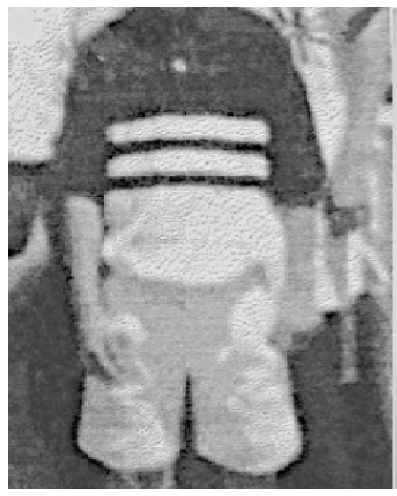

(a)

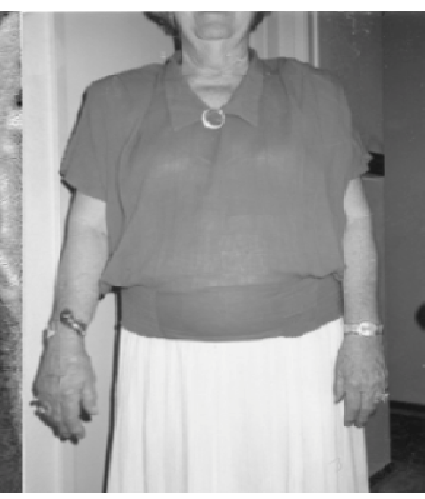

(b)
Figure 5.

Family Study D: (a) Generation 4 male diagnosed with LD demonstrating asymmetry of the right arm; (b) Generation 2 female with reported WLD demonstrating hyper-rotation of the right arm.

puter mouse upside down so as to operate a cursor on the computer in reverse. This was so the cursor would work in the opposite direction than her dominant left hand was moving. When she moved the mouse left, the cursor moved right, and when she moved downward the cursor moved upward (a computer instructor has informed us that he has had one or two students in each class who do this). After it was brought to her attention, by switching it to her right hand, she found she could directionally operate it correctly.

After accidentally injuring a finger on her dominant left hand, the subject found that, by using her right hand, she now could identify direction correctly. When using her dominant left hand, she never could remember how to use a key to lock or unlock a door correctly.

We worked with A.L. for approximately one year and she learned to use her right hand for other directional tasks. We also taught her to transfer a sense of direction from one hand to the other. We accomplished this by having her concentrate on how the movement would feel with the right hand while performing with the left. She also practiced her writing movement in the reverse direction from which she had always used. This is a clockwise top down direction movement, accomplished by tracing the letters with her non-writing hand; the hand, we had, found that she could use to tell direction. She then wrote over what she had learned with the writing hand. In time, she reported "feeling" the words, how they are seen and heard.

Eventually, she permanently shifted to a non-inverted and non-resting hand position by imitating the directional movement sensation of the right hand when writing out a word. What seemed to be of special help was turning the paper to the right instead of to the left, as she had grown accustomed to doing. After going to an exercise class for approximately one year, she told us that at first she became increasingly aware of how different the exercises felt between the two sides of her body. This was because the class consisted of many alternating of sides activities. When she was stretching and flexing, she had no trouble with her left arm, feet, toes, and leg.

It was an entirely different story with the right side of her body. She said that she had long thought that the limbs of her right side felt shorter than the left side of her body. A few other people from our large sample have also described this, claiming to have similar feelings in different parts of their bodies. In
A.L.'s case, the foot pointing and flexing exercises allowed her to see the problem differently. At first, as we had previously discussed with her, she believed that she suffered from a "displacement" of arm and leg location.

She told us: "The realization finally struck me that the leg did not feel shorter, it was that the right foot actually feels as if it is going in the opposite direction of how it is moved that causes the sensations" (i.e. if she points, her right toes down, they feel as if they are moving up). She also said: "My right leg also feels backwards. And, when my right arm is downward, it actually feels up. The hand and fingers on my right side however have normal sensations feeling up and down. The left arm felt like it was in a normal position while the hand felt backward.

Only after A.L. became aware of these sensory differences did we make a unique observation. It became apparent to us that her right arm can be easily turned in a different position than her left arm. It was as though the right arm was turned around. The muscle development was different and this arm hung quite naturally in this position. The fingers of both hands, however, tried to maintain their forward to backward position. The arms, in a relaxed hanging position, appeared normal forward to back. This unusual right arm position is achieved by stretching the arms down at each side.

Case 4. Had two sons (generation 4), both diagnosed with $\mathrm{LD}$ at five years of age. One of the two sons displayed the inverted bimanual movement behavior. At fourteen, he tested at 142 verbal and performance 121 full scale, 136 very superior on the Wechsler. He also had a history of ADHD behavior. The other son displayed a left bimanual top down rotation. This son, a good mathematician as evidenced by an 800 on his math SAT, always had trouble writing out the math problems and proving them on paper. Both sons, while graduating from college, experienced organizational difficulties as well as continued handwriting problems, a common symptom of WLD.

As the families from the first three cases we tested were ten members or fewer participants, the only family with a large enough population, Family Study D $(n=64)$ was used to make both physiological and behavioral comparisons. This was when specifically looking for suspected markers related to this type of learning difference. Four generations are represented.

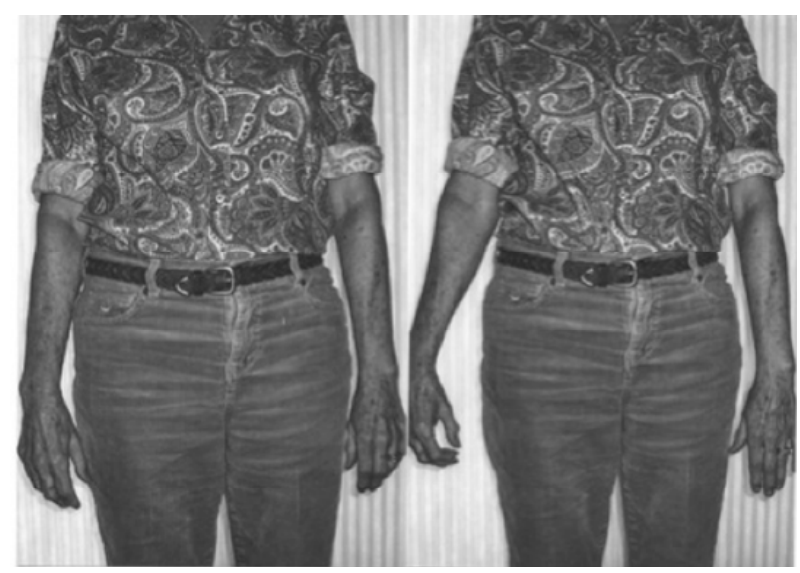

(a)

(b)

Figure 6.

Case 4. Family D; in relaxed and then stiffened positions demonstrating asymmetry. 
The biological members were separated from the non-biological members for comparison. The family members described as "biological" all have biological connections to the male or female in generation one. The non-biological group consisted of those persons ("in-laws") marrying into the family created by generation one.

The YGLD test results for Family D $(\mathrm{N}=64)$ are summarized in Tables 2 and 3.

\section{Test 1. Fine Motor Bimanual Rotation Test}

The first analysis compared the 3 groups on the 3 rotation preferences (Table 2). The chi square with $d f=4$ was $\chi^{2}=$ 38.47; $p<0.001$. This indicates that the distribution of rotation preference differs significantly from what would be expected by chance alone. Specifically, there is a strong tendency for "exclusive right or either top rotation" in both the Biological Non-WLD and Non-Biological Non-WLD groups. In contrast, there is a strong tendency for "inverted bimanual rotation" in the Biological WLD group. In this analysis there is a moderately strong association between group membership and rotation preference, Cramer's V $=0.55$.

\section{Test 2. Arm and Hand Positioning Test (For Asymmetry and/or an Arm Shifting Position)}

The first analysis compared the 3 groups on arm asymmetry (Table 3, middle column, shaded in gray) with the chi square goodness of fit test. The chi square with $d f=2$ was $\chi^{2}=12.18$; $p<0.01$. This indicates that the distribution of arm asymmetry differs significantly from what would be expected by chance alone. Specifically, most of the cases of asymmetry appeared in the Biological WLD group, with very few cases of asymmetry in either the Biological Non-WLD or non-biological, non-WLD groups.

\section{Test 3. Bimanual Arm Hand/Eye Dominance Test}

The second analysis compared the 3 groups on eye/arm sighting use (Table 3, right column, unshaded) with the chi square goodness of fit test. The chi square with $d f=2$ was $\chi^{2}=$ $5.64 ; p=0.06$. This result is just short of statistical significance. Although it is notable that there were no cases of right eye/arm or left eye/arm in the Biological WLD group, this finding does not differ from what could be expected by chance alone. Given the relatively rare occurrence of this phenomenon, resulting in low frequencies, a larger sample would be required to verify this finding.

The following is a summary of bio-behavioral identifications found in Family D:

1) WLD was not identified or reported in the non-biological group.

2) Eighty-seven percent $(n=27)$ of the biological, non-WLD group demonstrate "exclusive right or either top rotation".

$3)$ Eighty percent $(n=12)$ of the biological WLD demonstrated inverted rotation as well as any other method(s) as reported or as observed being used.

4) Seventy-seven percent $(n=14)$ of non-biological, nonWLD demonstrate "exclusive right or either top rotation".

5) Arm asymmetry and/or arm shift was identified in four percent $(n=49)$ of the non-WLD groups.

6) Arm asymmetry and/or arm shift was found in sixty-seven percent $(n=15)$ of the WLD group.

7) Twenty-two percent (11 of 49) biological and non-biological WLD subjects tested used their right arm hand/eye and their left arm hand/eye in both sighting positions.

8) There was no arm hand/eye lateral consistency in the WLD population.

The physical asymmetrical characteristics described have been observed in eleven of the $(n=46)$ other biologically related family members who we have tested. All but two were WLD identified. The reversed behavioral lid movement unscrewing test produced fourteen identifications in the biologically related group, of which all but two were identified as having written language problem history. The asymmetrical arm identification occurred in none of the other $(\mathrm{n}=17)$ nonbiologically related family members tested. The reversed behavioral lid unscrewing movement characteristic was observed in one subject in this group. No LD identification was reported

Table 2.

Number of subjects with type of rotation preference, $n$.

\begin{tabular}{cccc}
\hline \multirow{2}{*}{ Family D, family grouping $(\mathrm{N}=64)$} & \multicolumn{3}{c}{ Preferred behavioral marker } \\
\cline { 2 - 4 } & Inverted Bottom Rotation & Exclusive Left Top Rotation & Exclusive Right or Either Top Rotation \\
\hline Biological non-WLD $(\mathrm{n}=31)$ & 2 & 2 & 27 \\
Biological WLD $(\mathrm{n}=15)$ & 12 & 2 & 1 \\
Non-biological non-WLD $(\mathrm{n}=18)$ & 1 & 3 & 14 \\
\hline
\end{tabular}

Table 3.

Number of subjects with arm asymmetry and arm/hand/eye sighting consistency, $n$.

\begin{tabular}{ccc}
\hline \multirow{2}{*}{ Family D, family grouping $(\mathrm{N}=64)$} & \multicolumn{2}{c}{ Physical markers } \\
\cline { 2 - 3 } & Asymmetrical arms & Right arm, hand and eye Left arm, hand and eye use consistency \\
\hline Biological non-WLD $(\mathrm{n}=31)$ & 2 & 6 \\
Biological WLD $(\mathrm{n}=15)$ & 9 & 0 \\
Non-biological non-WLD $(\mathrm{n}=18)$ & 0 & 5 \\
\hline
\end{tabular}


in this non-biological member.

\section{Discussion}

Our data indicate that there is a significant correlation between reported written language learning difficulties and reversed bimanual movement behavior. Asymmetrical arms and other reversed physical characteristics have also been identified. The authors have previously reported evidence of a motoric link to written language disabilities and suggested that neural mechanisms for the control of timing precision and serial ordering in speech and language overlap extensively with neuronal processes for temporal organization of uni-manual and bimanual motor skills at a neuroanatomical, physiological, and behavioral level of description (Young \& Ginsburg, 1993).

Our present data indicate that there is a significant correlation between reported written language learning difficulties and inverted bi manual movement behavior and asymmetrical physical arm characteristics. A good example was found in case 4, "A.L." in Family 4. We theorize that the actual reason for AL being left handed was: that the left dominant arm, controlled the hand she first learned to write, however, as the left hand took control, it subsequently reverted to its naturally inverted sensing position. The asymmetry as shown in the right arm, we theorize, is a marker for the inverted sensation occurring in this region of her right arm.

By teaching this subject to straighten the left hand and teach her to the feel how her correctly sensing right hand felt the shape of letters and symbols, she was now able to correctly sense the feel of the shape of the symbols she was trying to learn to use. She too has expressed remarkable improvement in her written language abilities since this experimental remedial approach was implemented.

Due to our present small sample for arm hand/eye consistency, the data did not reach significance in Family D. However, it is notable that there were no cases of right arm hand/eye and left arm hand/eye consistency in the Biological WLD group. This finding does not differ from what could be expected by chance alone. Given the relatively rare occurrence of this phenomenon, resulting in low frequencies, a larger sample would be required to verify this finding.

We previously presented evidence for visual/motor dominance pattern differences between $\mathrm{LD}$ and non-LD, in which $4 \%$ of a population $(\mathrm{N}=1120)$ tested demonstrated right arm hand/eye and left arm hand/eye dominance consistency (Young $2006,2008)$. There was one case of diagnosed LD with this visual motor pattern out of the $(n=50)$ subjects found with this hand/eye pattern. He also was found to have the bi manual inverted rotation marker. This subject was a high achieving private middle school student scoring at the 99.9th national percentile rank on his Educational Records Bureau (ERB) total aptitude score. Using this measurement, the non-WLDs in the Family D population were almost at $22 \%$ representation. None of these consistent hand/eye dominance patterns were found in the WLD population.

Interestingly, the only two bimanual inverters in the biological non-WLD group both had this less common arm hand/eye dominance consistency. The mother of Case 2 also had this same arm hand/eye dominance pattern. She reported spelling problems, yet was a Harvard graduate. This may suggest a learning advantage with the biologically consistent right arm hand/eye and left arm hand/eye laterality dominance combina- tion.

Our one non biological family D MEMBER reporting inverted bimanual behavior had a right/left-right/right visual pattern. She did however report "messy" hand writing. There were no cases of LD reported out of the twenty-eight cases from our prior data base of $n=1120$ with this visual pattern. This is another example where arm eye/handedness may play an important part in better understanding learning disabilities.

Our few attempts at remediation by changing a hand position to sense a top down position when writing have shown to be successful. As the remedial experimentation was with a just a small number of participants, these results cannot be considered conclusive. However, the evidence does make it suggestive.

Although early academic skills would be most affected by RPS, each new learning process, such as moving into new symbolic information learning (e.g., different math subjects and foreign languages) would be consequently slowed down. This would put affected individuals at continual risk. We further suggest, the reversal sensation affecting movement behavior in RPS is a logical possibility and primary cause for certain ADHD behaviors identified with these learning difficulties.

None of the non-biologically related family members of the first generation $(n=17)$ were found to have a history of WLD, whereas $38 \%$ of the biologically connected part of the family group reported as LD. This would suggest a genetic connection. The father and his 3 sons in Family A displayed asymmetry, and bimanual inversion was found in all 5 of his children as well as himself. It has been recently reported to us that their 6th (male) child, who we did not test, also shares the characteristics along with an LD label. While ADHD was noted in many of these participants, no direct measurement of the label was attempted.

DNA testing of many of the WLD and non-WLD members was attempted and results were inconclusive. Unfortunately, when those studies were carried out we lacked the methods that are now available. It is our hope that in light of this continued research, that a DNA study will be reattempted for further characterization of RPS.

The majority of the RPS-affected cases that we have followed have managed to succeed in their various academic pursuits. If proper understanding and remedial help for those suspected to have this undiagnosed learning problem were administered, we suggest that far less stress for both students and teachers would result.

\section{Conclusion}

The evidence supports that both behavioral as well as physiccal markers for written learning difficulties possibly related to ADHD do exist. Aiding in the early identification of specific learning problems in such a way may lead to future successful remedial action. Ways to create a feeling of an interpretable, top down writing sensation for symbolic information would identifiably differ from one subject to another. After proper identification of where reversed sensations occur, a change of sidedness e.g. from right to left as well as left to right writing handedness is not as unthinkable as it sounds. In some cases just a hand repositioning has been found to be effective. Learning the feeling of a top/down directional sensation when writing would be important when learning and remembering symbolic information. Not correctly feeling what is seen and heard logically would be confusing and consequently disruptive to basic 
organization. Interceding once reversal of movement perception was identified and encouraging adoption of corrected movement repositioning might, especially in preschool and the early grades, greatly improve overall achievement and consequently eliminate further remedial effort. For the benefit of those with these unrecognized learning disadvantages, we are arguing for further exploration of a possibly efficient new way to both identify and treat RPS, an invisible contributor to both WLD as well as ADHD.

\section{Acknowledgement}

The authors would like to thank Chris Segrin, Ph.D., University of Arizona for assistance with statistical analysis and Penny Baron, M.S., for editorial assistance in the preparation of this manuscript.

\section{REFERENCES}

Adams, J., Gopher, D., \& Lintern, G. (1977). Effects of visual and proprioceptive feedback on motor learning. Journal of Motor Behavior, 9, 11-22.

Bradway, D. A. (2003). Behavioral genetic investigation into directional motor skills and reading disability. Unpublished Dissertation, University of Connecticut, Storrs.

Carter, B. F. (1977). Patterns of cerebral asymmetry in families affected by specific developmental dyslexia. Unpublished Dissertation, University of Connecticut, Storrs.

Carter, B. F., Ginsburg, B. E., \& Isaacs, S. (1982). Right-handed families: A genealogical rarity. Behavior Genetics, 23, 579.

Cook, A. S., \& Woollacot, M. H. (2006). Motor control: Translating research into clinical practice. Philadelphia, PA: Lippincott Williams \& Wilkins.

Educational Records Bureau (1982). The educational records bureau aptitude and achievement test. Level 4-Form C. Princeton, NJ: Educational Testing Service.

Ginsburg, B. E., Carter, B. F., \& Sadick, T. L. (1980). Patterns of laterality in children: Sinister effects of sinistrality in males. Behavior Genetics, 10, 494.

Ginsburg, B. E., Carter, B. F., \& Sadick, T. L. (1981). A genetic model of human laterality pattern. Behavior Genetics, 11, 600.

The Riverside Publishing Company, \& Houghton Mifflin Company (1993). Iowa Tests of Basic Skills.

Katusic, S. K., Colligan, R. C., Weaver, A. L., et al. (2009). The forgotten learning disability: Epidemiology of written language disorder in a population-based birth cohort (1976-1982), Rochester, Minnesota. Pediatrics, 123, 1306-1313. doi:10.1542/peds.2008-2098

Haager, D. (2007). Promises and cautions regarding using response to intervention with English language learners. Learning Disability Quarterly, 30, 213-218. doi:10.2307/30035565
LeDoux, J. E., Wilson, D. H., \& Gazzaniga, M. S. (1977). Manipulospatial aspects of cerebral lateralization Clues to the origin of lateralization. Neuropsychologia, 14, 743-750. doi:10.1016/0028-3932(77)90004-5

Longstaff, J. A. (2003). Model for practical kinesthesia. The Conference of the International Association for Dance Medicine \& Science (IADMS), London, UK, October.

Lyon, G. R. (1996). Learning disabilities. The Future of Children, 6, 54-76. doi: $10.2307 / 1602494$

Robertson, S. D. (2001). Development of bimanual skill: The search for stable patterns of coordination. Journal of Motor Behavior, 32, 114126. doi:10.1080/00222890109603144

Sadick, T. L., \& Ginsburg, B. E. (1978). The development of the lateral functions and reading ability. Cortex, 14, 3-11.

Young, R. A., \& Ginsburg, B. E. (1987, 2011). Young-Ginsburg lateral direction assessment and spatial test; "YGLD" Storrs, CT.

Young, R. A., \& Ginsburg, B. E. (1987). Genetic variations in motor and cognitive patterns associated with reading disabilities-Diagnosis and remediation. Behavior Genetics, 17, 644.

Young, R. A. (1989). Preferred directional rotational motor movement of the fingers and hands compared to reported receptive/expressive written language ability. Unpublished Thesis, University of Connecticut, Storrs.

Young, R. A., \& Ginsburg, B. E. (1993). The directional motor link in reading disabilities; in temporal information processing in the nervous system-With special reference to dyslexia and dysphasia. Annals of the New York Academy of Sciences, 682, 436-439. doi:10.1111/j.1749-6632.1993.tb23016.x

Young, R. A., \& Ginsburg, B. E. (1995). Visuo-motor patterns in dyslexia. Behavior Genetics, 25, 294.

Young, R. A. (2004). Laterality in kinesthetic rotation of movement orientation and visual dominance interaction as mediators of consciousness; Toward a science of consciousness. The Consciousness Conference at the University of Arizona, Tucson, AZ.

Young, R. A. (2006). Tested lateral visual and motor behavior interaction identify suspected aspects of Learning Disabilities. Behavior Genetics, 36, 952-990.

Young, R. A. (2008). Lost in Space-"Inverted Positioning Sensation" (IPS): As of yet unidentified contributing cause for ADHD and/or LD. The Consciousness Conference at the University of Arizona, Tucson, AZ, Abstract 121.

Wender, P. H., Wolf, L. E., \& Wasserstein, J. (2001) Adults with ADHD: An overview. Annals of the New York Academy of Sciences, 931, 1-16. doi:10.1111/j.1749-6632.2001.tb05770.x

Wechsler Intelligence Scale for Children-Revised, ed. (WISC-II) (1986).

Wolf, P. H., Melngallis, I., Obreon, M., \& Bedrosian, M. (1996) Family patterns of developmental dyslexia part III: Spelling errors as behavioral phenotype. American Journal of Medical Genetics, 67, 378-386. doi:10.1002/(SICI)1096-8628(19960726)67:4<378::AID-AJMG11> 3.0.CO;2-G 